\title{
FRENET FORMULAS FOR A GENERAL SUBSPACE OF A RIEMANN SPACE*
}

\author{
BY \\ E. H. CUTLER
}

Introduction. The Frenet formulas for a curve in ordinary space have been extended by Blaschke† to a curve in a Riemann space $V_{m}$. In $\$ \$ 1-4$ of the present paper it is shown that by utilizing a properly defined covariant differentiation similar formulas can be obtained for any subspace $V_{n}$ of a $V_{m} . \ddagger$ For a curve the curvatures are arbitrary functions of the parameter; for a general subspace the corresponding quantities are functions of the coordinates $x^{i}$ which must satisfy certain integrability conditions, the Gauss, Codazzi, Ricci equations. In $\$ 5$ a curve in the subspace is considered and Meusnier's Theorem extended, while in $\$ 6$ certain relations of $V_{n}$ to its osculating geodesic spaces are discussed.

1. Complete tensors and complete derivatives. Consider a Riemann space $V_{m}$ with definite fundamental tensor $a_{\alpha \beta}$, and let $V_{n}$ with fundamental tensor $g_{i j}$ be a subspace given by

$$
y^{\alpha}=y^{\alpha}\left(x^{1}, \cdots, x^{n}\right) \quad(\alpha=1, \cdots, m),
$$

where these, as all other functions, will be assumed analytic. Assume there is given at each point $P$ of $V_{n}$ a set of $n_{1}$ mutually perpendicular unit vectors $\zeta_{a_{1}} \mid \alpha$ in $V_{m}$ :

$$
\left.\left.a_{\alpha \beta} \zeta_{a_{1}}\right|^{\alpha} \zeta_{b_{1}}\right|^{\beta}=\delta_{a_{1} b_{1}} \quad\left(a_{1}, b_{1}=1, \cdots, n_{1}\right) .
$$

These vectors determine at $P$ an $n_{1}$-dimensional linear vector subspace of $V_{m}$, and any other set of $n_{1}$ mutually perpendicular unit vectors $\zeta_{a_{1}{ }^{\prime}}{ }^{\alpha}$ in this subspace is given by

$$
\left.\zeta_{a_{1}}\right|^{\alpha}=\left.t_{a_{1}}^{b_{1}} \zeta_{b_{1}}\right|^{\alpha}
$$

where

$$
t_{a_{1}}^{b_{1}} t_{c_{1}}^{b_{1}}=\delta_{a_{1} c_{1}} \S
$$

* Presented to the Society, February 28, 1931; received by the editors February 10, 1931.

$\dagger$ Mathematische Zeitschrift, vol. 6, pp. 94-99.

$\ddagger$ Since submission of the present paper there has appeared another on the same subject: J. A. Schouten and E. R. van Kampen, Eine Revision der Krummungstheorie, Mathematische Annalen, vol. 105, p. 144. These results were presented by Professor Schouten to the Society at its April meeting. See also Tucker, Generalized covariant differentiation, Annals of Mathematics, vol. 32, p. 451.

$\S$ Repeated indices are summed regardless of position. 
and conversely. We shall seek properties of this vector subspace which are independent of the choice of the $n_{1}$ vectors in it.

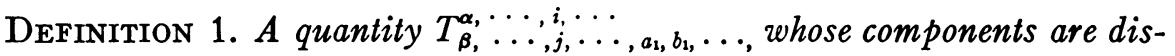
tinguished by any number of indices $\alpha, \beta, \cdots=1, \cdots, m$, and of $i, j, \ldots$ $=1, \cdots, n$, and of $a_{1}, b_{1}, \cdots=1, \cdots, n_{1}$, is a complete tensor if for a coordinate change in $V_{m}$ or $V_{n}$ it transforms as an ordinary tensor, and if for a change (1.3) in chosen system of the vectors $\left.\zeta_{a_{1}}\right|^{\alpha}$ it transforms as

$$
T_{\beta, \cdots, j, \cdots, a_{1}, b_{1}, \cdots}^{\alpha \alpha, \cdots, i, \cdots} t_{a_{1}}^{c_{1}} t_{b_{1}}^{d_{1}} \cdots T_{\beta, \cdots, j, \cdots, c_{1}, d_{1}, \cdots}^{\alpha, \cdots, i, \cdots}
$$

The sum or outer product of two complete tensors is a complete tensor, as is the contraction of a complete tensor contracted for a pair of indices of any of the three types.

Consider the covariant derivative defined by

$$
\begin{aligned}
& T_{\beta, \cdots, j, \cdots, a_{1}, \cdots ; k}^{\alpha, \cdots, i, \cdots}=\frac{\partial}{\partial x^{k}} T_{\beta, \cdots, i, \cdots, a_{1}, \cdots}^{\alpha, \cdots, i, \cdots}+\left\{\begin{array}{c}
i \\
k l
\end{array}\right\}_{0} T_{\beta, \cdots, i, \cdots, a_{1}, \cdots}^{\alpha, \cdots, l, \cdots}
\end{aligned}
$$

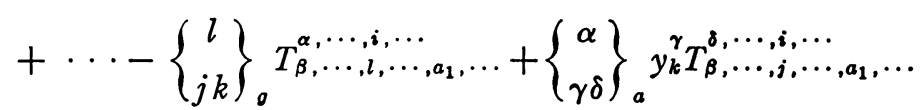

$$
\begin{aligned}
& +\cdots-\left\{\begin{array}{c}
\gamma \\
\beta \delta
\end{array}\right\}_{a}^{\delta} y_{k}^{\delta} T_{\gamma}^{\alpha, \cdots, i, \cdots, a_{1}, \cdots,}
\end{aligned}
$$

where $y_{i}{ }^{\alpha}$ means $\partial y^{\alpha} / \partial x^{i}$. We verify that under a change of coördinates either in $V_{m}$ or in $V_{n},\left(T_{\beta,}^{\alpha,}, \ldots, j, \cdots, a_{1}, \ldots ; k\right)$ transforms as a tensor, but under change (1.3) of $\left.\zeta_{a_{1}}\right|^{\alpha}$ we find that

$$
\begin{aligned}
T_{\beta, \cdots, j, \cdots, a_{1}, \cdots ; k}^{\alpha, \cdots, i, \cdots}=t_{a_{1}}^{c_{1}} \cdots & T_{\beta, \cdots, j, \cdots, c_{1}, \cdots ; k}^{\alpha, \cdots, i, \cdots} \\
& +\left(\frac{\partial}{\partial x^{k}} t_{a_{1}}^{c_{1}}\right) t_{b_{1}}^{d_{1}} \cdots T_{\beta, \cdots, j, \cdots, a_{1}, d_{1}, \cdots+\cdots}^{\alpha, \cdots, i, \cdots}
\end{aligned}
$$

Hence this covariant derivative is not itself a complete tensor.

The system of vectors $\left.\zeta_{a_{1}}\right|^{\alpha}$ is, by definition, a complete tensor; we consider its covariant derivative,

$$
\left.\zeta_{a_{1}}\right|_{; i} ^{\alpha}=\left.\frac{\partial}{\partial x^{i}} \zeta_{a_{1}}\right|^{\alpha}+\left.\left\{\begin{array}{c}
\alpha \\
\beta \gamma
\end{array}\right\}_{a}^{y_{i}^{\gamma} \zeta_{a_{1}}}\right|^{\beta} .
$$

In terms of this we define

$$
\left.\Gamma_{a_{1} b_{1}}\right|_{i}=\left.\left.a_{\alpha \beta} \zeta_{a_{1}}\right|^{\alpha} \zeta_{b_{1}}\right|_{; i} ^{\beta}
$$

By differentiating (1.2) we have 


$$
\left.\Gamma_{a_{1} b_{1}}\right|_{i}+\left.\Gamma_{b_{1} a_{1}}\right|_{i}=0
$$

and under a change (1.3) of $\left.\zeta_{a_{1}}\right|^{\alpha}$ we have

$$
\begin{gathered}
\left.\zeta_{b_{1}}^{\prime}\right|_{; i} ^{\alpha}=\left.t_{b_{1}}^{a_{1}} \zeta_{a_{1}}\right|_{; i} ^{\alpha}+\left.\zeta_{a_{1}}\right|^{\alpha} \frac{\partial}{\partial x^{i}} t_{b_{1}}^{a_{1}}, \\
\left.\Gamma_{a_{1} b_{1}}^{\prime}\right|_{i}=\left.t_{a_{1}}^{c_{1}} t_{b_{1}} \Gamma_{c_{1} d_{1}}\right|_{i}+t_{a_{1}}^{c_{1}} \frac{\partial}{\partial x^{i}}\left(t_{b_{1}}^{c_{1}}\right) ;
\end{gathered}
$$

solving this for $\left(\partial / \partial x^{i}\right)\left(t_{b_{1}}^{c_{1}}\right)$ and substituting in (1.7), we have

$$
T_{\beta, \cdots, j, \cdots, a_{1}, \cdots(k}^{\alpha, \cdots, i_{k}}=t_{a_{1}}^{b_{1}} \cdots T_{\beta, \cdots, i, \cdots, b_{1}, \cdots(k,}^{\alpha, \cdots, i, \cdots}
$$

where

$$
T_{\beta, \cdots, i, \cdots, a_{1}, \cdots(k}^{\alpha, \cdots, i, \cdots}=T_{\beta, \cdots, j, \cdots, a_{1}, \cdots ; k}^{\alpha, \cdots, i, \cdots}-\left.\Gamma_{c_{1} a_{1}}\right|_{k} T_{\beta, \cdots, j, \cdots, a_{1}, \cdots}^{\alpha, \cdots, i, \cdots}-\cdots
$$

Definttion 2. $T_{\beta}^{\alpha,} \ldots, i, \ldots, a_{1}, \ldots(k$ as defined by (1.6) and (1.13) is the complete covariant derivative of the complete tensor $T_{\beta}^{\alpha,}, \ldots, i, \ldots, a_{1}, \ldots$

THEOREM 1. The complete covariant derivative of a complete tensor is a complete tensor.

THEOREM 2. Complete differentiation obeys the ordinary rules of differentiation.

A complete tensor which we are especially interested in differentiating completely is $\zeta_{a_{1}} \mid \alpha$. We have

$$
\left.\zeta_{a_{1}}\right|_{(i} ^{\alpha}=\left.\zeta_{a_{1}}\right|_{; i} ^{\alpha}-\left.\left.\Gamma_{b_{1} a_{1}}\right|_{i} \zeta_{b_{1}}\right|^{\alpha} .
$$

Projecting this on $\left.\zeta_{c_{1}}\right|^{\alpha}$ we have by (1.9) and (1.2) that

$$
a_{\alpha \beta} \zeta_{a_{1}}\left|{ }_{i}^{\alpha} \zeta_{c 1}\right|^{\beta}=0 \text {. }
$$

2. Successive derived vector spaces.* As before we are given at each point of $V_{n}$ an $n_{1}$-dimensional vector space in $V_{m}$. In this we choose arbitrarily $n_{1}$ mutually perpendicular unit vectors $\left.\zeta_{a_{1}}\right|^{\alpha}, a_{1}=1, \cdots, n_{1}$. Consider $\left.\zeta_{a_{1}}\right|_{{ }^{\alpha} i}$ defined above. These are $n_{1} n$ vectors in $V_{m}$ which may or may not be independent. Since $\zeta_{a_{1}} \alpha^{\alpha}$ and $\left.\zeta_{a_{1}}\right|^{\alpha}{ }_{i}$ are complete tensors, we see that any vector $\eta^{\alpha}$ in $V_{m}$ dependent on them for one system of coördinates $\left(x^{i}\right)$ in $V_{n}$, and one system of vectors $\left.\zeta_{a_{1}}\right|^{\alpha}$, will be dependent on the corresponding vectors for any other systems. Hence $\zeta_{a_{1}} \mid \alpha$ and $\left.\zeta_{a_{1}}\right|^{\alpha}{ }_{(i}$ determine a unique vector space in $V_{m}$ at each point of $V_{n}$. Let $\left(n_{1}+n_{2}\right)$ be its dimensionality at

\footnotetext{
* See Struik, Mehrdimensionale Differentialgeometrie, p. 109.
} 
a general point of $V_{n}$; at special points it may be less. At such a general point of $V_{n}$ there is determined an $n_{2}$-dimensional vector space lying in the $\left(n_{1}+n_{2}\right)$ space and perpendicular to the original $n_{1}$-dimensional vector space. In this we choose $n_{2}$ mutually perpendicular unit vectors $\left.\zeta_{a_{2}}\right|^{\alpha}$ and proceed as before with the vectors $\left.\zeta_{a_{2}}\right|^{\alpha}$ defining $\left.\zeta_{a_{2}}\right|^{\alpha}{ }_{(i}$. These will with $\left.\zeta_{a_{1}}\right|^{\alpha}$ and $\left.\zeta_{a_{2}}\right|^{\alpha}$ determine a vector space of $\left(n_{1}+n_{2}+n_{3}\right)$ dimensions in which we choose $n_{3}$ vectors $\left.\zeta_{a_{3}}\right|^{\alpha}$, etc. We thus obtain a series of derived vector spaces each perpendicular to all the preceding.

Let $\left.\zeta_{a_{q}}\right|^{\alpha}$ be the last of these derived vector spaces. $\left.\zeta_{a_{q}}\right|^{\alpha}$ is the first set for which $\zeta_{a_{u}} \mid{ }^{\alpha}{ }_{i}$ are all dependent on $\zeta_{a_{v}} \mid \alpha$ for $v \leqq u$. In general it will happen that these exhaust the independent vectors of $V_{m}$, and $m=\sum_{u=1}^{q}\left(n_{u}\right)$. However, it may happen that there are $n_{q+1}$ further independent vectors at a general point of $V_{n}$. Choosing these as perpendicular to each other and to the preceding we write them $\left.\zeta_{a_{q+1}}\right|^{\alpha}$. These last vectors will be spoken of as residual rather than derived.

The vectors $\left.\zeta_{a_{u}}\right|^{\alpha}$ satisfy the relations

$$
\begin{aligned}
& \left.\left.a_{\alpha \beta} \zeta_{a_{u}}\right|^{\alpha} \zeta_{b_{u}}\right|^{\beta}=\delta a_{u} b_{u} \quad(u=1, \cdots,(q+1)), \\
& \left.\left.a_{\alpha \beta} \zeta_{a_{u}}\right|^{\alpha} \zeta_{b_{v}}\right|^{\beta}=0, u \neq v .
\end{aligned}
$$

Differentiating (2.2) completely,

$$
\left.\left.a_{\alpha \beta} \zeta_{a_{u}}\right|^{\alpha}{ }_{(i} \zeta_{b_{v}}\right|^{\beta}+\left.\left.a_{\alpha \beta} \zeta_{a_{u}}\right|^{\alpha} \zeta_{b_{v}}\right|_{(i} ^{\beta}=0 .
$$

By definition of the $(u+1)$ st vector space, $\left.\zeta_{a_{u+1}}\right|^{\alpha}$, it follows that $\left.\zeta_{a_{u}}\right|^{\alpha}{ }_{(i}$ is dependent on $\zeta_{a_{v}} \mid \alpha, v=1, \cdots,(u+1)$. Hence by (2.3) and (1.15) $\left.\zeta_{a_{u}}\right|^{\alpha}{ }_{(i}$ is dependent only on $\left.\zeta_{a_{u-1}}\right|^{\alpha}$ and $\left.\zeta_{a_{u+1}}\right|^{\alpha}$. Letting

$$
\left.\Omega a_{u+1} a_{u}\right|_{i}=\left.\left.a_{\alpha \beta} \zeta_{a_{u}}\right|^{\alpha}{ }_{i} \zeta_{a_{u+1}}\right|^{\beta}
$$

we have, by (2.3),

$$
\begin{gathered}
\left.\zeta_{a_{u}}\right|_{(i} ^{\alpha}=\Omega a_{u+1} a_{u}\left|{ }_{i} \zeta_{a_{u+1}}\right|^{\alpha}-\Omega a_{u} a_{u-1}\left|{ }_{i} \zeta_{a_{u-1}}\right|^{\alpha}, \\
u=1, \cdots,(q+1) \text {, where }\left.\Omega a_{u} a_{u-1}\right|_{i}=0, u=1 \text { or }(q+1) .
\end{gathered}
$$

3. Integrability conditions. We define $\left.\Gamma_{a_{u} b_{u}}\right|_{i j}$ by the equation

$$
\left.\Gamma_{a_{u} b_{u}}\right|_{i j}=\left.\Gamma_{a_{u} b_{u}}\right|_{j, i}-\left.\Gamma_{a_{u} b_{u}}\right|_{i, j}+\Gamma_{c_{u} a_{u}}\left|{ }_{j} \Gamma_{c_{u} b_{u}}\right|_{i}-\Gamma_{c_{u} a_{u}}\left|{ }_{i} \Gamma_{c_{u} b_{u}}\right|_{j} \text {. }
$$

From (1.11) we verify directly that $\left.\Gamma_{a u b u}\right|_{i j}$ is a complete tensor unlike $\left.\Gamma_{a_{u} b_{u}}\right|_{i}$ itself.

We now obtain the integrability conditions for complete differentiation. 
Differentiating (1.13) and then interchanging the order of differentiation and subtracting, we have

$$
\begin{aligned}
& T_{\beta, \cdots, i, \cdots, a_{u}, \cdots(h(l)}^{\alpha, \cdots, i, \cdots} T_{\beta, \cdots, i, \cdots, a_{u}, \cdots(l(h}^{\alpha, \cdots, i, \cdots}=\Gamma_{b_{u} a_{u}} \mid{ }_{h l} T_{\beta, \cdots, j, \cdots, b_{u}, \cdots}^{\alpha, \cdots, i, \cdots}
\end{aligned}
$$

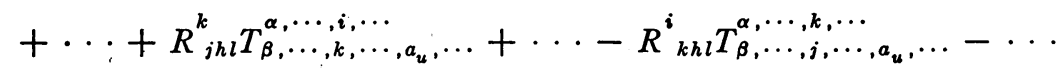

$$
\begin{aligned}
& +\bar{R}_{\beta \gamma \delta}^{e} y_{h}^{\gamma} y_{l}^{\delta} T_{\epsilon, \cdots, i, \cdots, a_{u}, \cdots}^{\alpha, \cdots, i, \cdots}+\cdots-\bar{R}_{\epsilon \gamma \delta}^{\alpha} y_{h}^{\gamma} y_{l}^{\delta} T_{\beta, \cdots, i, \cdots, a_{u}, \cdots}^{\epsilon, \cdots, \cdots,}
\end{aligned}
$$

Applying this condition to $\zeta_{a_{u}} \mid \alpha$ we have

$$
\left.\zeta_{a_{u}}\right|^{\alpha}{ }_{(i(j)}-\left.\zeta_{a_{u}}\right|^{\alpha}{ }_{(j i i}=-\left.\bar{R}_{\beta \gamma \delta}^{\alpha} \zeta_{a_{u}}\right|^{\beta} y_{i}^{\gamma} y_{j}^{\delta}+\Gamma_{a_{u} b_{u}}\left|{ }_{i j} \zeta_{b_{u}}\right|^{\alpha} .
$$

On the other hand using (2.5), we have

$$
\begin{aligned}
\left.\zeta_{a_{u}}\right|_{(i i j} ^{\alpha}= & \left.\Omega_{c_{u-1} b_{u-2}}\left|j \Omega_{a_{u} c_{u-1}}\right|_{i} \zeta_{b_{u-2}}\right|^{\alpha}-\Omega_{a_{u} c_{u-1}}\left|{ }_{i(j} \zeta_{c_{u-1}}\right|^{\alpha} \\
& -\left.\left[\left.\left.\Omega_{a_{u} b_{u-1}}\right|_{i} \Omega_{c_{u} b_{u-1}}\right|_{j}+\left.\left.\Omega_{b_{u+1} a_{u}}\right|_{i} \Omega_{b_{u+1} c_{u}}\right|_{j}\right]_{c_{u}}\right|^{\alpha} \\
& +\left.\left.\Omega_{b_{u+1} a_{u}}\right|_{i(i j} \zeta_{b_{u+1}}\right|^{\alpha}+\left.\Omega_{c_{u+2} b_{u+1}}\left|j \Omega_{b_{u+1} a_{u}}\right|_{i} \zeta_{c_{u+2}}\right|^{\alpha} .
\end{aligned}
$$

Substituting in (3.3) and projecting on the various $\left.\zeta_{a_{v}}\right|^{\alpha}$,

$$
\begin{aligned}
& \left.\Gamma_{a_{u} b_{u}}\right|_{i j}+\left(\left.\left.\Omega_{c_{u+1} b_{u}}\right|_{i} \Omega_{c_{u+1} a_{u}}\right|_{j}-\left.\left.\Omega_{c_{u+1} b_{u}}\right|_{j} \Omega_{c_{i+1} a_{u}}\right|_{i}\right) \\
& +\left(\left.\left.\Omega_{a_{u} c_{u-1}}\right|_{i} \Omega_{b_{u} c_{u-1}}\right|_{j}-\Omega_{a c_{u-1}}\left|{ }_{j} \Omega_{b_{u} c_{u-1}}\right|_{i}\right)=\left.\left.\bar{R}_{\alpha \beta \gamma \delta} \zeta_{a_{u}}\right|^{\beta} \zeta_{b_{u}}\right|^{\alpha} y_{i}^{\gamma} y_{j}^{\delta} \text {, } \\
& \left.\Omega_{a_{u} b_{u-1}}\right|_{i(j}-\left.\Omega_{a_{u} b_{u-1}}\right|_{j(i}=\left.\left.\bar{R}_{\alpha \beta \gamma \delta} \zeta_{b_{u-1}}\right|^{\alpha} \zeta_{a_{u}}\right|^{\beta} y_{i}^{\gamma} y_{j}^{\gamma}, \\
& \left.\left.\Omega_{a_{u} b_{u-1}}\right|_{i} \Omega_{b_{u-1} c_{u-2}}\right|_{j}-\left.\left.\Omega_{a_{u} b_{u-1}}\right|_{j} \Omega_{b_{u-1} c_{u-2}}\right|_{i}=\left.\left.\bar{R}_{\alpha \beta \gamma \delta} \zeta_{u_{u-2}}\right|^{\alpha} \zeta_{a_{u}}\right|^{\beta} y_{i}^{\gamma} y_{j}^{\gamma}, \\
& \left.\left.\bar{R}_{\alpha \beta \gamma \delta} \zeta_{a_{u}}\right|^{\alpha} \zeta_{b_{v}}\right|^{\beta} y_{i} y_{j}^{\delta}=0, \quad|u-v|>2 .
\end{aligned}
$$

4. Frenet and Gauss formulas. We will now and for the rest of the paper take our original $n_{1}$-dimensional vector space as the tangent vector space to $V_{n}$ in $V_{m}$. Then $n_{1}=n$,

$$
\begin{aligned}
\left.\zeta_{a_{1}}\right|^{\alpha} & =\left.\zeta_{a_{1}}\right|^{i} y_{i}^{\alpha}, \\
\left.\left.a_{\alpha \beta} \zeta_{a_{1}}\right|^{\alpha} \zeta_{b_{1}}\right|^{\beta} & =\left.\left.g_{i j} \zeta_{a_{1}}\right|^{i} \zeta_{b_{1}}\right|^{i}=\delta_{a_{1} b_{1}} .
\end{aligned}
$$

The vectors $\left.\zeta_{a_{1}}\right|^{i}$ are vectors of an orthogonal $n$-uple in $V_{n}$. The vectors $\left.\zeta_{a_{v}}\right|^{\alpha}$ for $v>1$ are all normal to $V_{n}$.

We verify that $y_{i}{ }^{\alpha},\left.\zeta_{a_{1}}\right|^{i}$ and $\left.\zeta_{a_{1}}\right|_{i}$ are complete tensors and that they satisfy 


$$
\begin{aligned}
\left.\zeta_{a_{1}}\right|_{i} & =\left.a_{\alpha \beta} y_{i}^{\alpha} \zeta_{a_{1}}\right|^{\beta}, \\
y_{i}^{\alpha} & =\left.\left.\zeta_{a_{1}}\right|^{\alpha} \zeta_{a_{1}}\right|_{i} .
\end{aligned}
$$

Since $y_{i}{ }^{\alpha}$ is a complete tensor we can differentiate completely

$$
y_{i(j}^{\alpha}=\frac{\partial^{2} y^{\alpha}}{\partial x^{i} \partial x^{j}}+\left\{\begin{array}{c}
\alpha \\
\beta \gamma
\end{array}\right\}_{a}^{y_{i}^{\beta} y_{j}^{\gamma}}-\left\{\begin{array}{c}
h \\
i j
\end{array}\right\}_{0}^{y_{h}^{\alpha}=y_{j(i}^{\alpha} .}
$$

Differentiating completely the equation

$$
g_{i j}=a_{\alpha \beta} y_{i}^{\alpha} y_{j}^{\beta},
$$

we have by a cyclic permutation of the indices and (4.5) that

$$
a_{\alpha \beta} y_{i}^{\alpha} y_{j(h}^{\beta}=0 .
$$

Differentiating (4.4) we have

$$
\underset{y_{i(i}}{\alpha}=\left.\left.\zeta_{a_{1}}\right|_{(i} ^{\alpha} \zeta_{a_{1}}\right|_{i}+\left.\left.\zeta_{a_{1}}\right|^{\alpha} \zeta_{a_{1}}\right|_{i(j} .
$$

By (4.6) and (4.5) we have

$$
\begin{gathered}
\left.\zeta_{a_{1}}\right|_{i(j}=0 \text { or } y_{i(j}^{\alpha}=\left.\left.\zeta_{a_{1}}\right|_{i \zeta} \zeta_{a_{1}}\right|_{(i,} ^{\alpha}, \\
\left.\left.\zeta_{a_{1}}\right|_{i} \zeta_{a_{1}}\right|_{(i} ^{\alpha}-\left.\left.\zeta_{a_{1}}\right|_{j} \zeta_{a_{1}}\right|_{(i} ^{\alpha}=0 .
\end{gathered}
$$

This latter is a set of $\frac{1}{2} n(n-1)$ vector relations connecting the $n^{2}$ vectors $\left.\zeta_{a_{1}}\right|_{(i .}$. The number of independent vectors is at most $\frac{1}{2} n(n+1)$, and we have $n_{2} \leqq \frac{1}{2} n(n+1)$.

By (1.9) and (4.6) we have

$$
\begin{aligned}
\left.\Gamma_{a_{1} b_{1}}\right|_{i} & =\left.a_{\alpha \beta} y_{l}^{\alpha} \zeta_{a_{1}}\right|^{l}\left(\left.y_{h}^{\alpha} \zeta_{b_{1}}\right|^{h}\right)_{i i}=\left.\left.g_{l h} \zeta_{a 1}\right|^{l} \zeta_{b_{1}}\right|_{i i} ^{h} \\
\left.\Gamma_{a_{1} b_{1}}\right|_{i j} & =\left.g_{l h} \zeta_{a_{1}}\right|^{l}\left(\left.\zeta_{b_{1}}\right|^{h}{ }_{, i, i}-\left.\zeta_{b_{1}}\right|_{, i, j} ^{h}\right)=\left.\left.\zeta_{a_{1}}\right|^{l} \zeta_{b_{1}}\right|^{h} R_{l h i j} .
\end{aligned}
$$

Equation (3.5) thus reduces for $u=1$ to an equivalent of the Gauss equation for $V_{n}$ in $V_{m}$, and the equations (3.5)-(3.8) are, as a set, equivalent to the ordinary Gauss, Codazzi, Ricci equations for $V_{n}$ in $V_{m}$. Whenever, as now, the $\zeta_{a_{1}}{ }^{\alpha}$ are the tangents to the $V_{n}$, equations (2.5) will be referred to as the Frenet formulas for $V_{n}$ in $V_{m}$. In justification of this consider a curve $V_{1}$. We can choose the arc-length as the coördinate $x^{1}$ and then $g_{11}=0$ and $\left\{\begin{array}{l}1 \\ 11\end{array}\right\}=0$. There will be just one vector tangent to the curve and just one in each of the

${ }^{*}$ In case there is only one derived vector space the present work is equivalent to that of Weyl, Mathematische Zeitschrift, vol. 12, pp. 154-160, and that of R. Lagrange, Thesis, Paris, 1923, chapter 5 . 
derived vector spaces. Hence by (1.10) $\left.\Gamma_{a_{u} b_{u}}\right|_{i}=0$ and the complete derivative reduces to

and we can write

$$
\left.\frac{d}{d s} \zeta_{a_{u}}\right|^{\alpha}+\left\{\begin{array}{c}
\alpha \\
\beta \gamma
\end{array}\right\} \zeta_{a} a_{a_{u}}^{\beta} \frac{d y^{\gamma}}{d s},
$$

$$
\left.\frac{d}{d s} \zeta_{u}\right|^{\alpha}+\left.\left\{\begin{array}{c}
\alpha \\
\beta \gamma
\end{array}\right\}_{a} \zeta_{u}\right|^{\beta} \frac{d y^{\gamma}}{d s}=-\left.\Omega_{u, u-1} \zeta_{u-1}\right|^{\alpha}+\left.\Omega_{u+1, u} \zeta_{u+1}\right|^{\alpha},
$$

which is precisely the Frenet equation where $\left(1 / \rho_{u}\right)=\Omega_{u, u-1}$.

5. A curve $V_{1}$ in $V_{n}$ in $V_{m}$. Assume we have given a curve $V_{1}$ in $V_{n}$ by

$$
x^{i}=x^{i}(s) \text {. }
$$

Then by (1.1) we have $V_{1}$ given in $V_{m}$ by

$$
y^{\alpha}=y^{\alpha}(s) \text {. }
$$

Let $\left.\xi_{1}\right|^{\alpha}, \cdots,\left.\xi_{m}\right|^{\alpha}$ be the $m$ associate vectors of (5.2) in $V_{m}$, and let $\left.\phi_{1}\right|^{i}, \cdots,\left.\phi_{n}\right|^{i}$ be the $n$ associate vectors of (5.1) in $V_{n}$. Let $\left.\lambda_{u}\right|^{\alpha}$ and $\left.\eta_{u}\right|^{i}$ be the normalized vectors corresponding to $\left.\xi_{u}\right|^{\alpha}$ and $\left.\phi_{u}\right|^{i}$ respectively. Then we have

$$
\left.\xi_{u}\right|_{, \beta} ^{\alpha} \frac{d y^{\beta}}{d s}=\left.\xi_{u+1}\right|^{\alpha},\left.\phi_{u}\right|^{i}, \frac{d x^{j}}{d s}=\left.\phi_{u+1}\right|^{i} .
$$

This covariant differentiation may be replaced by complete differentiation of $\left.\xi_{u}\right|^{\alpha}$ or $\left.\phi_{u}\right|^{i}$, it being understood that the subscript $u$ is not an index but a part of the symbol. Then

$$
\begin{aligned}
& \left.\xi_{u}\right|_{, \beta} ^{\alpha} \frac{d y^{\beta}}{d s}=\left.\xi_{u}\right|_{{ }_{\beta}} ^{\alpha} y_{i}^{\beta} \frac{d x^{i}}{d s}=\left.\xi_{u}\right|_{(i} ^{\alpha} \frac{d x^{i}}{d s}, \\
& \left.\phi_{u}\right|_{, j} ^{i} \frac{d x_{j}}{d s}=\left.\phi_{u}\right|_{{ }_{j}^{i} \frac{d x^{j}}{d s}} ^{i} .
\end{aligned}
$$

Moreover we have

$$
\left.\xi_{1}\right|^{\alpha}=\frac{d y^{\alpha}}{d s}=y_{i}^{\alpha} \frac{d x^{i}}{d s} .
$$

Differentiating this completely, using the Frenet formulas for $V_{n}$ in $V_{m}$,

$$
\begin{aligned}
\left.\xi_{2}\right|^{\alpha}= & \left.\xi_{1}\right|_{(j} ^{\alpha} \frac{d x^{j}}{d s}=\left(\left.\left.\left.\zeta_{a_{1}}\right|^{\alpha} \zeta_{a_{1}}\right|_{i} \phi_{1}\right|^{i}\right)_{(j} \frac{d x^{j}}{d s} \\
= & \left.\zeta_{a_{1}}\right|^{\alpha}\left(\left.\left.\zeta_{a_{1}}\right|_{i} \phi_{2}\right|^{i}\right)+\left.\zeta_{a_{2}}\right|^{\alpha}\left(\left.\left.\left.\left.\Omega_{a_{2} a_{1}}\right|_{j} \zeta_{a_{1}}\right|_{i} \phi_{1}\right|^{i} \phi_{1}\right|^{j}\right), \\
\left.(5.5) \quad \xi_{3}\right|^{\alpha}= & \left.\zeta_{a_{1}}\right|^{\alpha}\left(\left.\left.\left.\left.\left.\left.\Omega_{a_{2} a_{1}}\right|_{j} \Omega_{a_{2} b_{1}}\right|_{i} \zeta_{b_{1}}\right|_{k} \phi_{1}\right|^{i} \phi_{1}\right|^{j} \phi_{1}\right|^{k}+\left.\left.\zeta_{a_{1}}\right|_{i} \phi_{3}\right|^{i}\right) \\
& +\left.\zeta_{a_{2}}\right|^{\alpha}\left(\left.\left.\left.\left.\left.\Omega_{a_{2} a_{1}}\right|_{j(k} \zeta_{a_{1}}\right|_{i} \phi_{1}\right|^{i} \phi_{1}\right|^{i} \phi_{1}\right|^{k}+\left.\left.\left.\left.3 \Omega_{a_{2} a_{1}}\right|_{i} \zeta_{a_{1}}\right|_{j} \phi_{1}\right|^{i} \phi_{2}\right|^{j}\right) \\
& +\left.\zeta_{a_{3}}\right|^{\alpha}\left(\left.\left.\left.\left.\left.\left.\Omega_{a_{3} a_{3}}\right|_{i} \Omega_{a_{2} a_{1}}\right|_{j} \zeta_{a_{1}}\right|_{k} \phi_{1}\right|^{i} \phi_{1}\right|^{i} \phi_{1}\right|^{k}\right),
\end{aligned}
$$

and in general we have that $\left.\xi_{u}\right|^{\alpha}$ is dependent on $\left.\zeta_{a_{1}}\right|^{\alpha}, \ldots,\left.\zeta_{a_{u}}\right|^{\alpha}$. 
THEOREM 3. Given a $V_{1}$ in a $V_{n}$ in a $V_{m}$, then the uth osculating vector space of $V_{1}$ in $V_{m}$ is contained in the uth osculating vector space of $V_{n}$ in $V_{m}$.

$\left.\xi_{u}\right|^{\alpha}$ may also be expressed by repeated application of the Frenet formulas for $V_{1}$ in $V_{m}$. Thus we have

$$
\begin{aligned}
& \left.\xi_{1}\right|^{\alpha}=\left.\lambda_{1}\right|^{\alpha}, \\
& \left.\xi_{2}\right|^{\alpha}=\left.\left(1 / \rho_{1}\right) \lambda_{2}\right|^{\alpha}, \\
& \left.\xi_{2}\right|^{\alpha}=-\left.\left(1 / \rho_{1}\right)^{2} \lambda_{1}\right|^{\alpha}+\left.\frac{d}{d s}\left(1 / \rho_{1}\right) \lambda_{2}\right|^{\alpha}+\left.\left(1 /\left(\rho_{1} \rho_{2}\right)\right) \lambda_{3}\right|^{\alpha} .
\end{aligned}
$$

If $V_{n}$ in $V_{m}$ is known and if the vectors $\left.\lambda_{v}\right|^{\alpha}, v \leqq u$, of a curve are known then the quantities $\left(1 / \rho_{v}\right)$ and $\left(d^{r} / d s^{r}\right)\left(1 / \rho_{s}\right)$ entering into $\left.\xi_{v}\right|^{\alpha}, v \leqq u$, are determined. This follows from a comparison of (5.5) and (5.6). The coefficient of $\left.\lambda_{1}\right|^{\alpha}$ in $\left.\xi_{1}\right|^{\alpha}$ is known, being 1 . Assume the coefficients in the first $v$ equations (5.6) known and also $\left.\phi_{r}\right|^{i}, r<v$. Then $\left.\phi_{v}\right|^{i}$ is determined since $\left.\xi_{v}\right|^{\alpha}$ is given by (5.6), and in (5.5) $\left.\phi_{v}\right|^{i}$ will be the only unknown in the formula for $\left.\xi_{v}\right|^{\alpha}$. Then by (5.5) the projection of $\left.\xi_{v+1}\right|^{\alpha}$ on $\left.\zeta_{a_{v+1}}\right|^{\alpha}$ is known; the only term in (5.6) having such a projection is the last. Equating we have

$$
\begin{aligned}
& \left(1 /\left(\rho_{1} \rho_{2} \cdots \rho_{v+1}\right)\right)\left(a_{\alpha \beta} \lambda_{v+1}\left|\alpha \zeta_{a_{v+1}}\right|{ }^{\beta}\right) \\
& \quad=\left.\left.\Omega_{a_{v+1} a_{v}}{ }_{i} \Omega_{a_{v} a_{v-1}}{ }_{j} \cdots \Omega_{a_{2} a_{1}}\left|{ }_{k} \zeta_{a_{1}}\right|{ }_{l} \phi_{1}\right|^{i} \cdots \phi_{1}\right|^{\imath} .
\end{aligned}
$$

This determines $\left(1 / \rho_{v+1}\right)$ assuming that none of the preceding curvatures were zero. Next projecting on $\zeta_{a_{v}} \mid{ }^{\alpha}$ we determine $(d / d s)\left(1 / \rho_{v}\right)$, and so on. Only one previously undetermined quantity occurs in each projection and so can be determined. Proceeding by successive steps we show that $\left(d^{r} / d s^{r}\right)\left(1 / \rho_{s}\right)$, $r \geqq 0, s>0, r+s \leqq u$, and $\left.\phi_{v}\right|^{i}, v \leqq u$, are determined by the $\left.\lambda_{v}\right|^{\alpha}$ of a curve $v \leqq u$. The $\left.\lambda_{v}\right|^{\alpha}$ must however be the actual $\left.\lambda_{v}\right|^{\alpha}$ of some curve in $V_{n}$ in order that the conditions be compatible. For instance the component of $\left.\lambda_{v+1}\right|^{\alpha}$ normal to the vth osculating space of $v_{n}$ is determined except for magnitude by (5.7). For this reason we state the resulting theorem in the form

Theorem 4. Through a point $P$ given two curves lying in $V_{n}$ in $V_{m}$, and given further that, for a number $u$,

(a) the osculating vector spaces $\left.\zeta_{a_{1}}\right|^{\alpha}, \cdots,\left.\zeta_{a_{u}}\right|^{\alpha}$ of $V_{n}$ exist;

(b) the vectors $\left.\lambda_{1}\right|^{\alpha}, \cdots,\left.\lambda_{u}\right|^{\alpha}$ of the two curves as curves of $V_{m}$ are the same at $P$;

(c) the common tangent to the two curves is such that

$$
\left.\left.\left.\left.\left.\left.\Omega_{a_{u} a_{u-1}}\right|_{i} \Omega_{a_{u-1} a_{u-2}}\right|_{j} \cdots \Omega_{a_{2} a_{1}}\right|_{k} \zeta_{a_{1}}\right|_{l} \phi_{1}\right|^{\imath} \cdots \phi_{1}\right|^{i}
$$

is not zero for all $a_{u}$; then the curratures $\left(1 / \rho_{v}\right), v \leqq u$, and their derivatives 
$\left(d^{r} / d s^{r}\right)\left(1 / \rho_{s}\right), r+s \leqq u$, and the $V_{n}$ associate vectors of the two curves are the same at $P$.

For $u=1$ this theorem reduces to an equivalent of Meusnier's theorem.

The case of $V_{1}$ in $V_{n}$ in $V_{m}$ is a special case of the more general problem of $V_{l}$ in $V_{n}$ in $V_{m}$. For this more general problem it follows, just as for a curve, that the first $u$ osculating spaces of $V_{l}$ in $V_{m}$ are contained in the first $u$ osculating vector spaces of $V_{n}$ in $V_{m}$. A formula analogous to (5.7) holds for the more general problem, but it cannot always be solved for the curvature tensor which replaces the $\left(1 / \rho_{u}\right)$. Hence we cannot proceed in this case to the extension of the theorem above.

6. Residual normals. The residual normals, $\left.\zeta_{a_{q+1}}\right|^{\alpha}$, were defined as normals not in derived vector spaces of any order; they were characterized by the equation

$$
\left.\Omega_{a_{q+1} a_{q}}\right|_{i}=0
$$

Hence by (2.5)

$$
\left.\zeta_{a_{q+1}}\right|_{(i} ^{\alpha}=0 .
$$

Equation (6.1) expresses a condition in terms of the $\left.\Omega_{a_{q+1} a_{q}}\right|_{i}$ that the set of $n_{q+1}$ normals $\left.\zeta_{a_{q+1}}\right|^{\alpha}$ be residual; conditions that $V_{n}$ in $V_{m}$ possess $n_{q+1}$ residual normals may also be expressed in terms of the ordinary theory of subspaces where the normals are not separated into successive sets.

Following the notation of Eisenhart* we denote a set of $(m-n)$ normals by $\xi_{\sigma} \mid{ }^{\alpha}, \sigma=1, \cdots,(m-n)$, and define

$$
\begin{aligned}
\left.\mu_{\tau \sigma}\right|_{i} & =\left.a_{\alpha \beta} \xi_{\tau}\right|^{\alpha}\left[\left.\xi_{\sigma}\right|_{, i} ^{\beta}+\left.\left\{\begin{array}{c}
\beta \\
\gamma \delta
\end{array}\right\}_{a} y_{i}^{\gamma} \xi_{\sigma}\right|^{\delta}\right], \\
\left.\Omega_{\sigma}\right|_{i j} & =\left.a_{\alpha \beta} \xi_{\sigma}\right|^{\alpha}\left[\begin{array}{c}
\beta \\
y_{i, j}
\end{array}+\left\{\begin{array}{c}
\beta \\
\gamma \delta
\end{array}\right\}_{a} y_{i}^{\gamma} y_{i}^{\delta}\right] \\
& =-a_{\alpha \beta} y_{i}^{\alpha}\left[\left.\xi_{\sigma}\right|_{, i} ^{\beta}+\left.\left\{\begin{array}{c}
\beta \\
\gamma \delta
\end{array}\right\}_{a} y_{i}^{\gamma} \xi_{\sigma}\right|^{\delta}\right] .
\end{aligned}
$$

We now choose as one particular set $\left.\xi_{\sigma}\right|^{\alpha}$ the set $\left.\zeta_{a q+1}\right|^{\alpha},\left.\zeta_{a_{q}}\right|^{\alpha}, \cdots,\left.\zeta_{a_{1}}\right|^{\alpha}$ in that order. Writing (6.2) in the form

$$
\left.\zeta_{a_{q+1}}\right|_{, i} ^{\alpha}+\left.\left\{\begin{array}{c}
\alpha \\
\beta \gamma
\end{array}\right\}{ }_{a}^{\beta} \zeta_{i} \zeta_{a_{q+1}}\right|^{\gamma}-\Gamma_{b_{q+1} a_{q+1}}\left|{ }_{i} \zeta_{b_{q+1}}\right|^{\alpha}=0
$$

* Riemannian Geometry, chapter IV. 
we see from (6.3) and (6.4) that for this set of normals

$$
\begin{array}{lr}
\left.\mu_{+\sigma}\right|_{i}=0 & \left(\sigma=1, \cdots, n_{q+1} ; \tau=n_{q+1}, \cdots,(m-n)\right), \\
\left.\Omega_{\sigma}\right|_{i j}=0 & \left(\sigma=1, \cdots, n_{q+1}\right) .
\end{array}
$$

Equations (6.5), (6.6) for some normal system are sufficient as well as necessary for the existence of $n_{q+1}$ residual normals. For from (6.6) it follows that the first derived vector space is in space of $\left.\xi_{\tau}\right|^{\alpha}, \tau>n_{q+1}$, and by (6.5) this follows for the further derived spaces.

If we change to another set of normals

$$
\begin{aligned}
\left.\xi_{\sigma}^{\prime}\right|^{\alpha} & =\left.t_{\sigma}{ }^{\top} \xi_{\tau}\right|^{\alpha} \quad \quad(\tau, \sigma=1, \cdots,(m-n)), \\
\left.\Omega_{\sigma}^{\prime}\right|_{i j} & =\left.t_{\sigma}{ }^{\top} \Omega_{\tau}\right|_{i j},
\end{aligned}
$$

and $\left.\Omega_{\sigma}\right|_{i j}$ is normal covariant unlike $\left.\mu_{\tau \sigma}\right|_{i}$. We define the complete derivative of a normal covariant quantity in the way indicated by the example

$$
\left.\Omega_{\sigma}\right|_{i j(k}=\left.\Omega_{\sigma}\right|_{i j, k}-\left.\left.\mu_{\tau \sigma}\right|_{k} \Omega_{\tau}\right|_{i j} \quad(\tau, \sigma=1, \cdots,(m-n)) .
$$

The complete derivative is itself normal covariant and differentiation obeys the ordinary rules.*

If (6.6) holds for one normal system, then for any other there are $n_{q+1}$ independent sets of solutions of

$$
\left.\eta_{\sigma} \Omega_{\sigma}\right|_{i j}=0 \text {. }
$$

If $\eta_{\sigma}$ is such a solution both $\eta_{\sigma}$ and $\eta_{\sigma(k}$ are normal covariant, and for our special normal system we verify that $\eta_{\sigma(k}=0$. Hence for any normal system we have

$$
\eta_{\sigma(k}=0 \text {. }
$$

Differentiating (6.8) we have, by (6.9),

$$
\begin{aligned}
\left.\eta_{\sigma} \Omega_{\sigma}\right|_{i j(k} & =0, \\
\left.\eta_{\sigma} \Omega_{\sigma}\right|_{i j(k(l)} & =0,
\end{aligned}
$$

A necessary condition that $V_{n}$ in $V_{m}$ possess $n_{q+1}$ residual normals is that equations (6.8) and (6.10) admit $n_{q+1}$ independent solutions.

Conversely, assume that the first $Q$ equations of (6.8) and (6.10) admit a complete set of $n_{q+1}$ solutions which satisfy the $(Q+1)$ st. Taking the normals corresponding to these solutions as the first $n_{q+1}$ reference normals $\left.\xi_{\sigma}\right|^{\alpha}, \sigma=1, \cdots, n_{q+1}$, we can show that (6.5) and (6.6) are satisfied.

* See Weyl and Lagrange, loc. cit. 
ThEOREM 5. A necessary and sufficient condition that $V_{n}$ in $V_{m}$ possess $n_{q+1}$ residual normals is that (6.8) and the first $Q$ equations of (6.10) admit a complete set of $n_{q+1}$ solutions which also satisfy the $(Q+1)$ st.

The simplest example of a space $V_{n}$ possessing residual normals is a totally geodesic space $T_{n}$ in $V_{m}$. For such a subspace the $\left.\Omega_{\sigma}\right|_{i j}$ vanish identically and conditions (6.8), (6.10) are satisfied by all normals, which are therefore all residual. Conversely, if a $V_{n}$ in $V_{m}$ possess $(m-n)$ residual normals, by (6.8) we see that $\left.\Omega_{\sigma}\right|_{i j}=0$ and $V_{n}$ is a $T_{n}$ of $V_{m}$.

THEOREM 6. A necessary and sufficient condition that $a V_{n}$ in $V_{m}$ possess $(m-n)$ residual normals is that it be a totally geodesic subspace of $V_{m}$.

The sufficient part generalizes as follows:

Theorem 7. If $V_{n}$ is any subspace of a totally geodesic subspace $T_{N}$ of $V_{m}$, then the normals to $T_{N}$ will be residual normals of $V_{n}$ in $V_{m}$.

This could be proved directly by choosing Riemannian coördinates and direct computation; it follows also from Theorem 3 . The converse of this theorem is not true; that is, the existence of residual normals does not imply that $V_{n}$ lies in a totally geodesic subspace of $V_{m}$. For example, it can be shown that there always exist curves with only one derived normal, the principal normal; and in fact that such curves exist through any given point with any given pair of perpendicular vectors as tangent and principal normal. But the point and pair of vectors may be such that the geodesic surface in $V_{m}$ determined by them is not totally geodesic.

Another question concerning a $V_{n}$ with residual normals which arises is that of the relation of the $V_{n}$ to its complete osculating geodesic space. For curves the author has shown that if the complete osculating geodesic space is totally geodesic the curve lies in it. The proof by means of Riemannian coördinates and direct computation holds for the general subspace.

Definition. The uth osculating geodesic space of $V_{n}$ in $V_{m}$ at $P$ is made up of $V_{m}$ geodesics through $P$ in directions dependent on $\left.\zeta_{a_{1}}\right|^{\alpha}, \cdots,\left.\zeta_{a_{u}}\right|^{\alpha}$. The complete osculating geodesic space at $P$ is made up of geodesics through $P$ perpendicular to all the residual normals at $P$.

Theorem 8. If the complete osculating geodesic space of $V_{n}$ in $V_{m}$ at $P$ is totally geodesic then $V_{n}$ lies in it.

Theorem 9. A necessary and sufficient condition that $a V_{n}$ in $a V_{m}$ of constant curvature possess $(m-N)$ independent residual normals is that it be a subspace of a geodesic subspace $G_{N}$ of $V_{m}$. 
Theorem 10. A necessary and sufficient condition that a $V_{n}$ in $a V_{m}$ of constant curvature lie in an $N$-dimensional geodesic subspace is that (6.8) and (6.10) admit $(m-N)$ solutions in the usual sense.

Other theorems on the relations of a curve to its osculating geodesic spaces may be extended to the present case. The proofs are the same as for the curve.

TheOREM 11. Let $P^{\prime}:\left(x^{i}+\Delta x^{i}\right)$ be a point of $V_{n}$ near $P:\left(x^{i}\right)$. The principal parts of the infinitesimal distances of $P^{\prime}$ from the tangent $G_{n}$ and the osculating $G_{n+n_{2}}$ at $P$ are given by

$$
\begin{aligned}
& d^{2}=\frac{1}{4} \sum_{a_{2}}\left(\left.\left.\Omega_{a 2 a_{1}}\right|_{i} \zeta_{a_{1}}\right|_{j} \Delta x^{i} \Delta x^{j}\right)^{2}+\cdots \\
& d^{2}=\frac{1}{36} \sum_{a_{3}}\left(\left.\left.\left.\Omega_{a_{3} a_{2}}\right|_{i} \Omega_{a_{2} a_{1}}\right|_{j} \zeta_{a_{1}}\right|_{k} \Delta x^{i} \Delta x^{i} \Delta x^{k}\right)^{2}+\cdots,
\end{aligned}
$$

except where these expressions vanish.

Theorem 12. If the uth osculating geodesic space $G_{N}$ of $V_{n}$ in $V_{m}$ at a general point $P$ is totally geodesic, then the principal part of the distance of $P^{\prime}:\left(x^{i}+\Delta x^{i}\right)$ from it is in general

$$
d^{2}=\sum_{a_{u+1}}\left(\left.\left.\left.\left.\Omega_{a_{u+1} a_{u}}\right|_{i} \Omega_{a_{u} a_{u-1}}\right|_{j} \cdots \Omega_{a_{2 a 1} a_{1}}\right|_{k} \zeta_{a_{1}}\right|_{l} \Delta x^{i} \cdots \Delta x^{l}\right)^{2} /[(u+2) !]^{2}+\cdots
$$

TheOREM 13. If $V_{n}^{\prime}$ is the projection of $V_{n}$ in $V_{m}$ on its osculating geodesic space of $\left(n+n_{2}\right)$ or $\left(n+n_{2}+n_{3}\right)$ dimensions, then for properly chosen reference systems we have at $P$

$$
\begin{array}{ll}
\left.\Omega_{a_{2} a_{1}}\right|_{i}=\left.\Omega_{a_{2} a_{1}}^{\prime}\right|_{i} & \text { (either case), } \\
\left.\Omega_{a_{3} a_{2}}\right|_{i}=\left.\Omega_{a_{3} a_{3}}^{\prime}\right|_{i} & \text { (second case) } .
\end{array}
$$

THEOREM 14. If $V_{N}$, the uth osculating geodesic space of $V_{n}$ in $V_{m}$ at $P\left(N=n+n_{2}+\cdots+n_{u}\right)$, is totally geodesic, and if $V_{n}^{\prime}$ is the projection of $V_{n}$ on it, then for properly chosen reference systems

$$
\left.\Omega_{a_{v} a_{v-1}}\right|_{i}=\left.\Omega_{a_{v} a_{v-1}}^{\prime}\right|_{i} \quad(v=2, \cdots, u) .
$$

LEHGH UNIVERSITY, Bethlehem, Pa. 